\title{
The portable automatic test instrument design and implementation using in radar
}

\author{
Daoju Duan ${ }^{1, a}$, Zhonghua Zhang ${ }^{1, b}$, Rui Li ${ }^{1, c}$ and Ajian Zhang ${ }^{1, d}$ \\ ${ }^{1}$ Fire Adjustment Radar Faculty of Ordnance Military School of Wuhan, Wuhan, Hubei, 430075, \\ China

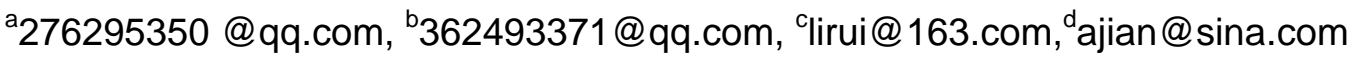

Keywords: ARM9; automatic test; radar maintenance; portable equipment.

\begin{abstract}
The main matter of the artificial test is the slow speed in the process of radar equipment maintenance, combining with the characteristic of radar fault self checking circuit, so we designed the portable radar automatic test instrument based on embedded system, this instrument used the ARM9 motherboard as its main controller. We developed the interface circuit, data acquisition module and the automatic test software, and we realized the function of Once Connected, Repeated Measurement in the process of circuit parameter test. The apparatus improves the efficiency of repair test, and has the certain promotion value.
\end{abstract}

\section{Introduction}

At present, the radar maintainability design, widely used the BIT technology, and set up the circuit module, and provided artificial test interface used to diagnose fault and test parameter for the radar equipment maintenance supply method. When the radar equipment appears serious fault, the technical staff can test the signals in the artificial interface using the multimeter or oscilloscope, but can only test a signal once time. In order to observe the variation characteristics of radar circuit working state during the transition, they need frequent switching machine, observation and comparison, the repair efficiency changed very low. Automatic test system can quickly measure the technical parameters of radar through certain preprogrammed, but now ATS commonly use GPIB, VXI, PXI bus, with Large size and high price, and cannot provide on-site technical maintenance service[1].

If we can development a kind of portable automatic test equipment realizing the function of Once Connected, Repeated Measurement, automatic test the information of current, voltage, waveform through the preset test interface of radar, we would greatly improve the efficiency of fault repair through configuring this device in the field level. Because of the need of this portable ATS, we developed the portable radar automatic test equipment which would help the technicians quickly locate the fault component.

\section{System Solutions}

The portable ATE[2] is used to measure the detection interface parameters in a radar, including analog and digital signal which have voltage amplitude:-10 +10V and frequency under 10Mhz.The method of measure is that the ATE load pre programmed software, connecting the ATE with each detection interface in radar subsystem using adapters, then the interface signals access the measurement circuit successively through the switch array of the identifiable pin interface, finally displaying the signal parameters on the monitor of the ATE. The overall structure of the ATE includes the adapter module, measurement circuit module, embedded main control board, display control module and power supply module, and the test software developed for ATE. The system solutions are illustrated in Fig 1.

Not only the Connection mode of the signals in each radar subsystem test interface is great difference but also the contact pin of Input signal under test in the ATE are fixed, which make us design different adapter cables of each test interface in order to connect the signals waiting for 
measure to the ATE interface contact pin, making the test circuit measure. Each subsystem corresponds to an own adapter cable, so on the basis of making a synthesis of each interface cable features, we share three cables: 8 channel, 12 channel and 24 channel. By means of this three adapter cables, we can measure the signals on the 10 interfaces of radar.

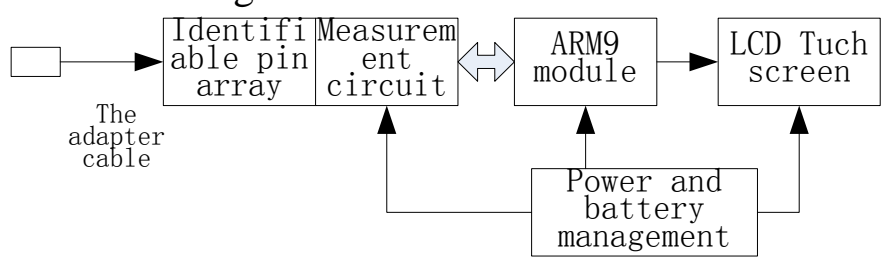

Figure 1.System Solutions

Interface array module connecting the adapter cable through drawing out measure pins is used to connect the subsystem test signals of radar to the ATE. The measurement circuit module is used to measure the analogue signals through time-sharing, to measure frequency and duty ratio of the pulse signal, and to measure the digital signals is inputted to the relevant ports of embedded motherboard to be measured. The embedded main control motherboard is the core part of the whole equipment, and the microcontroller unit (MUC) adopt ARM chip. This chip with fast running speed is rich in on-chip resources, and has a certain amount of storage space. The MCU with working procedure is used to initialize and calibrate the measurement circuits, then measure signals parameters according to the testing procedures, finally send the measurement results to displayed on the monitor. The display and control module is the LCD display and function key aside. We use the touch screen to enhance the ability of human-computer interaction. The DC power using lithium battery power supply is used to supply the required voltage of the ATE.

\section{The main composition of hardware}

The interface design. The interface circuit module adopts 4 CD4067 chips to realize more about 64 inputs and 1 output[3]. The chip CD4067 is equivalent to 16 choose 1 switch, which is enable decided by the microcontroller output address code. The contact interface pins include two parts: multiple pins socket and coaxial pins socket. The multiple pins part adopts the universal standard connector produced by VPC. This interface is use to measure the low frequency signals such as AC or DC voltage through the ATE software which defined the interface pin character signals from the radar test interface. Coaxial socket which like the multiple pins socket is used to measure the high frequency signals such as the frequency, pulse width of the pulse signal.

The measurement circuit. The measurement circuit is realized by using the method of time division multiplexing to measure each signal one by one, which is used for the detection of voltage and waveform characteristics, as shown in figure 2.

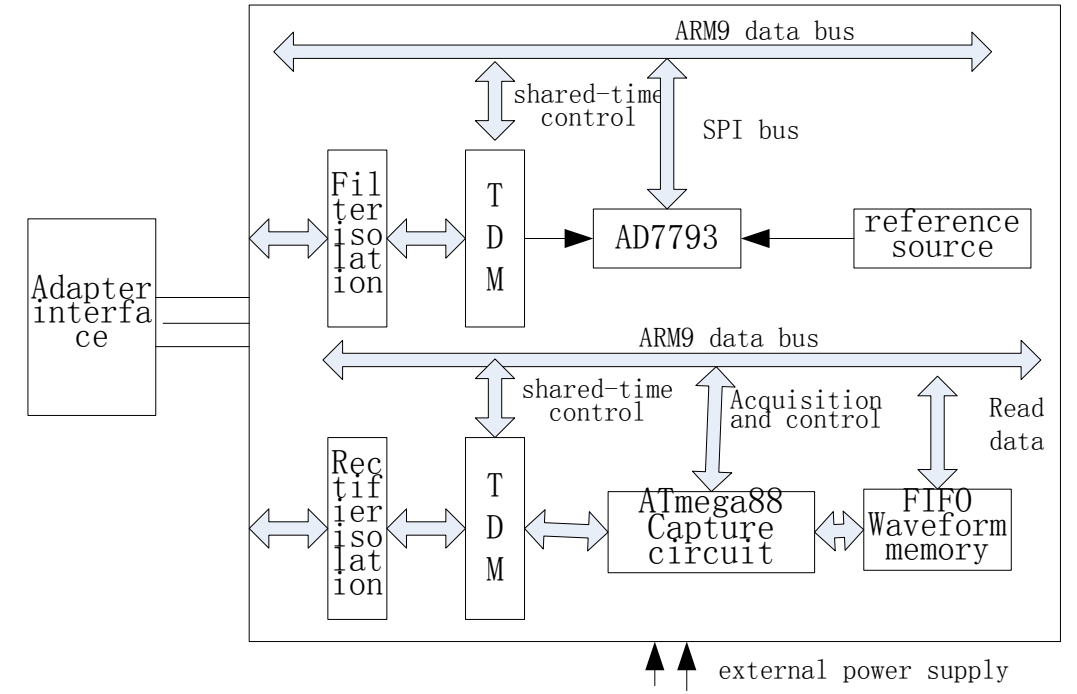

Figure 2. the design module of measurement circuit 
We use the chip ad7793 to measure the analog signal. The AD7793 are low power, low noise, complete analog front ends for high precision measurement applications. It contains a low noise 16-/24-bit $\sum-\triangle \mathrm{ADC}$ with three differential analog inputs. The on-chip low noise instrumentation amplifier means that signals of small amplitude can be interfaced directly to the ADC. With a gain setting of 64,the RMS noise is $40 \mathrm{nV}$ when the update rate equals $4.17 \mathrm{~Hz}$. The devices can be operated with either the internal clock or an external clock. The output data rate from the parts is software-programmable and can be varied from $4.17 \mathrm{~Hz}$ to $470 \mathrm{~Hz}$. In order to meet the requirement of the measurement precision, the AD7793 use an external reference voltage source, and connect with the MCU using the SPI bus, which can preset the communication rate, sampled on the rising edge etc.

We realized the feature of pulse frequency and duty cycle measurement with the input capture of the AVR single - chip microcomputer. Because the AVR SCM usually has two inputs capture pin, multiple input signals is measured adopting time division multiplexing technology which is a way that we realized the use of capture pin according to the time sequence by time division switching circuit. Because the pulse waveforms have jump edge, in order not to cause interference to the follow-up circuit, isolation circuit before and after is used to isolate the input signal. In our design solution, the system uses optical isolation technology to overcome the crosstalk problems in the current products. In addition, in order to overcome the problems such as the parasitic inductance generating line, and to achieve the purpose of accurate measurement of waveform parameters, it is necessary to adopt the Schmidt trigger circuit to shape the waveform. The main control computer adopts data bus to trigger AVR microcomputer to capture the signal in when and which fashion. Once the microcomputer captures the rising edge of the signals, the microcomputer counts it in the period of the smallest unit time of $0.1 \mathrm{~s}$, and stop when the ascendant edge come again, and the count value is the cycle of the signal. At the same time the sampled waveform is sent to the FIFO memory cache. Finally ARM chip read the waveform data in a parallel bus to memory and display it on the monitor.

Control and display.This portable radar ATE based on embedded system adopts the hardware of ARM framework. We the ARM9 SPI bus to access the A/D converter, acquire and store data, even to control the other integrated chip via the parallel bus. In the course of system integration, we use the ARM bus to control the time division switch and AVR single chip microcomputer. The ARM9 chip with rich on-chip resources adopt memory and clock chip with I2C interface bus to clock and memory the important data when the system works. We use I2S external power amplifier and loudspeaker to alarm and point out the mistaken operation. When the ARM9 chip connect with the other module circuit, we adapt SPI bus to connect the main controller card with the chip AD7793, and use the LCD controller interface from the ARM card. The monitor is a 5.7 inch color STNLCD, which use the D/A interface from ARM9 to control the the screen brightness. in the end, we draw forth one parallel bus to be used for the control bus to control two time division switch ,AVR MCU and to read the waveform.

\section{Software Design}

Embedded Operating System.The embedded operating system $\mu \mathrm{C} / \mathrm{OS}$ - II is a free pay, priority based, preemptive, cutting open source code and multitask real-time operating system, and is suitable for our system being proved by both the theory and practice. It only is a real-time operating system kernel, and it is necessary to combine the application program and the underlying hardware into a real-time kernel system. Therefore, our program developers must build ourselves real-time operating system based on this real-time kernel. The kernel cannot provide standard API functions, so we developed the application program based on this kernel[4].

Program design.It is the key of realizing portable ATE design to program the Once Connected, Repeated Measurement software. Depending on the hardware this software in test program order automatically measure all the specified parameters from every measured interface of the radar, and in the course of measurement the interface pins only are connected once. The main function of the 
software includes two parts: data acquisition and data processing. Data collection procedures used to coordinate and control switch network to the establish the measurement channel between the measured object and the measuring circuit ,and used to control and set the measurement circuit parameters at the same time. Finally this procedure reads the original data to the ARM memory. The data processing program according to the current system state, such as: the switch state, measurement error and other factors, select suitable calibration data to revise the original data, then according to the specified format process and display the necessary data. The software model is shown in figure 3.

When the system software is startup, it is the first step for the user to enter the system management module to configure the system parameters. The system software initialize procedure based on the user permissions, and prompts the user select suitable test adapter cable connector according to the different measured interface. After the test procedures is loaded, the system runs the test procedure and restore the test result. Finally the test result has been used to be analyzed and transmitted to the fault diagnosis software module. The fault diagnosis module determine where the fault happened, and help the user to remove failures. In order to facilitate the user, system has designed two kinds of measurement model. One is the automatic test mode. The system software run the test procedures in turn, and guides the operator to automatically complete the test task. The operator can pause, continue and cancel the whole taste. The other is manual mode that the operator can measure one or several parameters as it is needed, and can measure once again [5].

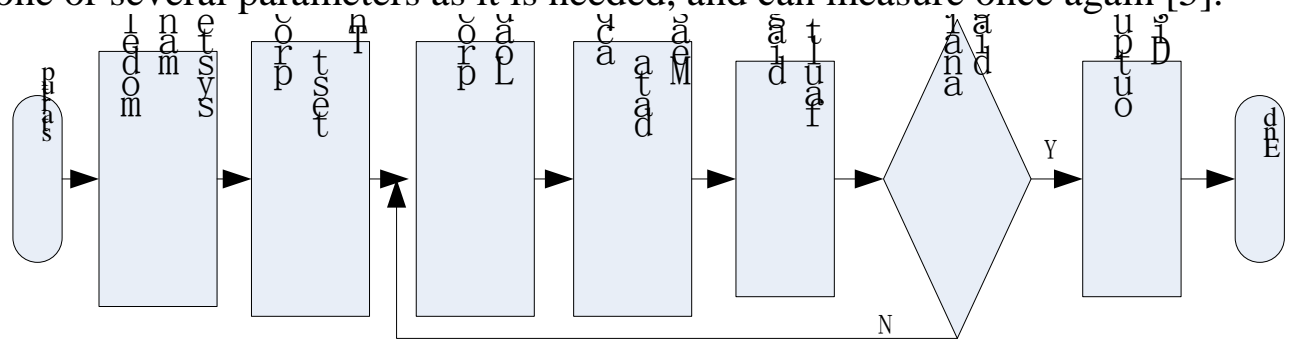

Fig 3. software design

\section{Conclusions}

The portable ATE adopting embedded technical platform can be used to measure the maintenance data and diagnose faults through designing interface and measurement circuit and developing ATE software. When the ATE is deployed in the basic level, repair guarantee means will change more and more rich and perfect, and improve the automation and intelligent level of equipment repair. If we develop different interface adapters and test procedures modules, this ATE will be applied to others radar maintenance support, so this ATE has certain value of popularization.

\section{Reference}

[1] Xiao Mingqing, Hu Gang-lei, Wang Yi. Automatic Test Survey[M]. Beijing: National Defense Industry Press, 2012.08.

[2] Li Li-gong, Nian Fu-shun, Wang Hou-jun. Modern electronic measuring technology [M]. Beijing: National Defense Industry Press, 2008.01.

[3] Wang Shouquan, Liu Wenbo, Guo Weibo. Design and Implement of Handed Radar ATE [J]. Measurement \& Control Technology, 2010,30(1):67-70.

[4] Gao Junyao Zhao Jia. Development of Display and Control System Based on $\mu$ C/OS- II [J]. Microcontrollers \& Embedded System, 2009, (12): 43-45.

[5] Duan Daoju, Zhang Yongzhen. Developing and Applying of Radar Maintenance Diagnosis Platform Based on Virtual Technology [J]. Electronic Engineer,2008.34 (4):1-3. 